\title{
Appropriate antibiotic therapy in critically ill patients
}

\author{
Filippo Pieralli, Antonio Mancini, Andrea Crociani \\ Intermediate Care Unit, Careggi University Hospital, Firenze, Italy
}

\begin{abstract}
Severe sepsis and septic shock are leading causes of morbidity and mortality in critically ill patients in and outside Intensive Care Units. Early hemodynamic and respiratory support, along with prompt appropriate antimicrobial therapy and source control of the infectious process are cornerstone management strategies to improve survival. Antimicrobial therapy should be as much appropriate as possible, since inappropriate initial antimicrobial therapy is associated with poorer outcome in different clinical settings. When prescribing antibiotic therapy, drug's characteristics, along with dosing, pharmacokinetics, and pharmacodynamic properties related to the drug and to the clinical scenario should be well kept in mind in order to achieve maximal success.
\end{abstract}

\section{Introduction}

Severe sepsis and septic shock are leading causes of morbidity and mortality in critically ill patients in and outside Intensive Care Units. There is an increasing evidence that patients are frequently cared in general wards (not considering if appropriate or not) even in the presence of severe sepsis and septic shock..$^{1,2}$

Early hemodynamic and respiratory support, along with prompt appropriate antimicrobial therapy and source control of the infectious process are cornerstone management strategies to reduce mortality.,4

Antimicrobial therapy should be as much appropriate as possible, since inappropriate initial antimicrobial therapy is associated with poorer outcome in different clinical settings. In patients with septic shock inappropriate antibiotic therapy, which has been reported up to $20 \%$ of cases, is associated with a dramatic reduction in the probability of survival. ${ }^{5}$

In this chapter, we will review the basis of empir-

Correspondence: Filippo Pieralli, Subintensiva di Medicina, Azienda Ospedaliero-Universitaria Careggi, Largo Brambilla 3, 50139 Firenze, Italy.

Tel./Fax: +39.055357845

E-mail: filippopieralli@gmail.com

Key words: Antibiotic; sepsis; critical care; infection.

Received for publication: 6 October 2016.

Accepted for publication: 20 October 2016

This work is licensed under a Creative Commons Attribution NonCommercial 4.0 License (CC BY-NC 4.0).

(C) Copyright F. Pieralli et al., 2016

Licensee PAGEPress, Italy

Italian Journal of Medicine 2016; 10:275-281

doi:10.4081/itjm.2016.792 ical antimicrobial therapy in the critically ill septic patient, revising an approach focused on some topics.

\section{Choosing the right antibiotic dosing strategy: pharmacokinetic and pharmacodynamic considerations}

Many pathophysiological changes can occur in critically ill patients and can complicate antibiotic dosing. Knowledge of the pharmacokinetic and pharmacodynamic properties of antibiotics is essential for choosing dosing regimens. Changes in volume of distribution $(\mathrm{Vd})$ and clearance $(\mathrm{CL})$ of antibiotics may affect the antibiotic concentration at the target site and have been extensively described in septic patients (Figure 1).

Different antibiotic classes have been shown to have different killing characteristics on bacteria (Figure 1 and Table 1).

Optimizing the dosing regimen is important for maximizing patient's response and for minimizing the development of antibiotic resistance.

Based on their killing characteristics antibiotics are distinguished in time-dependent, concentration-dependent and concentration-dependent antibiotics with time dependence (Figure 2).

Time-dependent antibiotics (e.g., $\beta$-lactams), achieve their maximal activity when unbound plasma concentrations of the drug are maintained above the minimum inhibitory concentration (MIC) for a defined fraction of the dosing interval ( $f \mathrm{~T} / \mathrm{MIC}$ ). On the other hand, concentration-dependent antibiotics (e.g., aminoglycosides), offer their optimal killing activity when maximal concentration, represented by its ratio with the MIC (Cmax/MIC), is achieved. A third category of drugs shows a concentration-dependent with time dependence profile, which implies that a ratio between the unbound area under the curve (AUC) and 
the MIC of the bacteria ( $f$ AUC0-24/MIC) correlates with optimal activity. ${ }^{6}$

$\beta$-lactams, the most widely used antibiotics, are time-dependent antibiotics. Many studies have shown that $\beta$-lactam concentrations are inadequate in septic patients (compared to non-critically ill patients) when standard dosage regimens are administered specially when dealing with difficult-to-treat infections such as those caused by Pseudomonas aeruginosa. ${ }^{7,8}$

Current evidence from clinical studies and Monte Carlo simulations (which is a mathematical method by which through repeated random sampling numerical results are obtained) suggest that prolonged infusion for $\beta$-lactams can increase the achievement of the pharmacodynamics target associated with efficacy and may improve patient outcomes.

\section{Pharmacokinetic changes observed in critically ill patients}

Changes in pharmacokinetics in the critically ill patients are due both to drug and disease factors. From a drug perspective, the hydrophilicity and lipophilicity of the molecule will influence $\mathrm{Vd}$ and $\mathrm{CL}$ of a drug. ${ }^{9}$

$\beta$-lactams, amynoglycosides, glycopetides are hydrophilic agents with renal clearance and $\mathrm{Vd}$ affected by the total body water (TBW) volume, while fluoroquinolones, macrolides, oxazolidinones, glycylcy- clines, metronidazole are lipophilic and therefore less affected by TBW. Daptomycin is amphiphilic (hydrophilic and lipophilic), then with a more complex behavior, however from a practical point of view it should be regarded as hydrophilic. ${ }^{10}$

\section{Changes in volume of distribution}

The pathogenesis of infections in critically ill patients is highly complex. In addition to fluid resuscitation and vasopressors, endotoxins from bacteria or fungi may cause endothelial dysfunction via production of endogenous mediators, leading to maldistribution of blood flow, endothelial damage, and increased capillary permeability.

As a consequence, capillary leakage syndrome determines fluids shift from the intravascular compartment to the interstitial space, increasing the Vd of hydrophilic drugs which, in turn, decrease their plasma unbound drug concentration. ${ }^{11}$

\section{Hypoalbuminemia}

Protein binding is a factor that may influence the $\mathrm{Vd}$ and CL of many antibiotics. Hypoalbuminemic states (a common finding in the critically ill) can result in a higher unbound concentration that has up to $100 \%$ increased CL and $90 \%$ greater $\mathrm{Vd} .{ }^{12,13}$

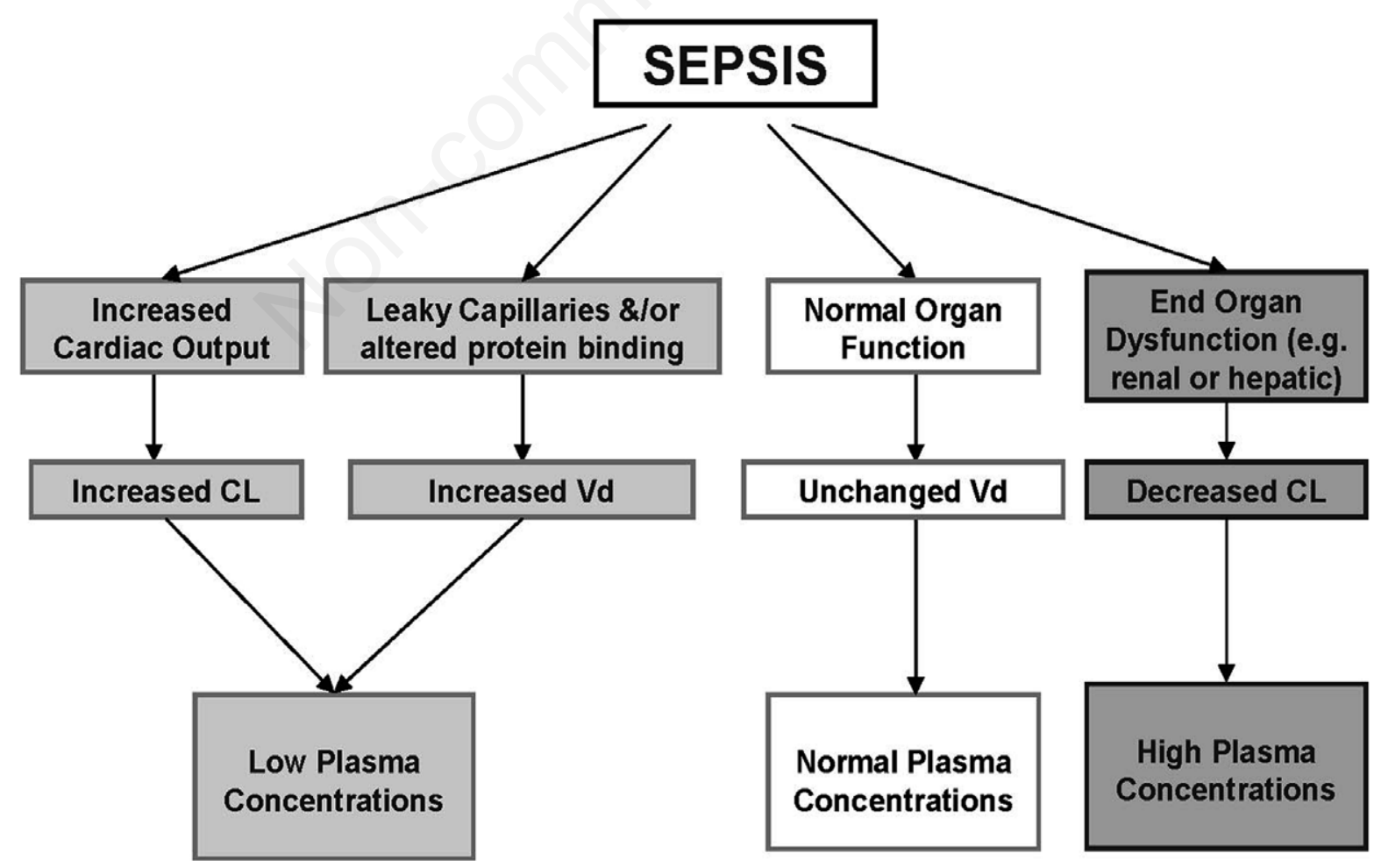

Figure 1. Plasma concentrations of antibacterial drugs according to the pathophysiology of sepsis. CL, clearance; Vd, volume of distribution. 


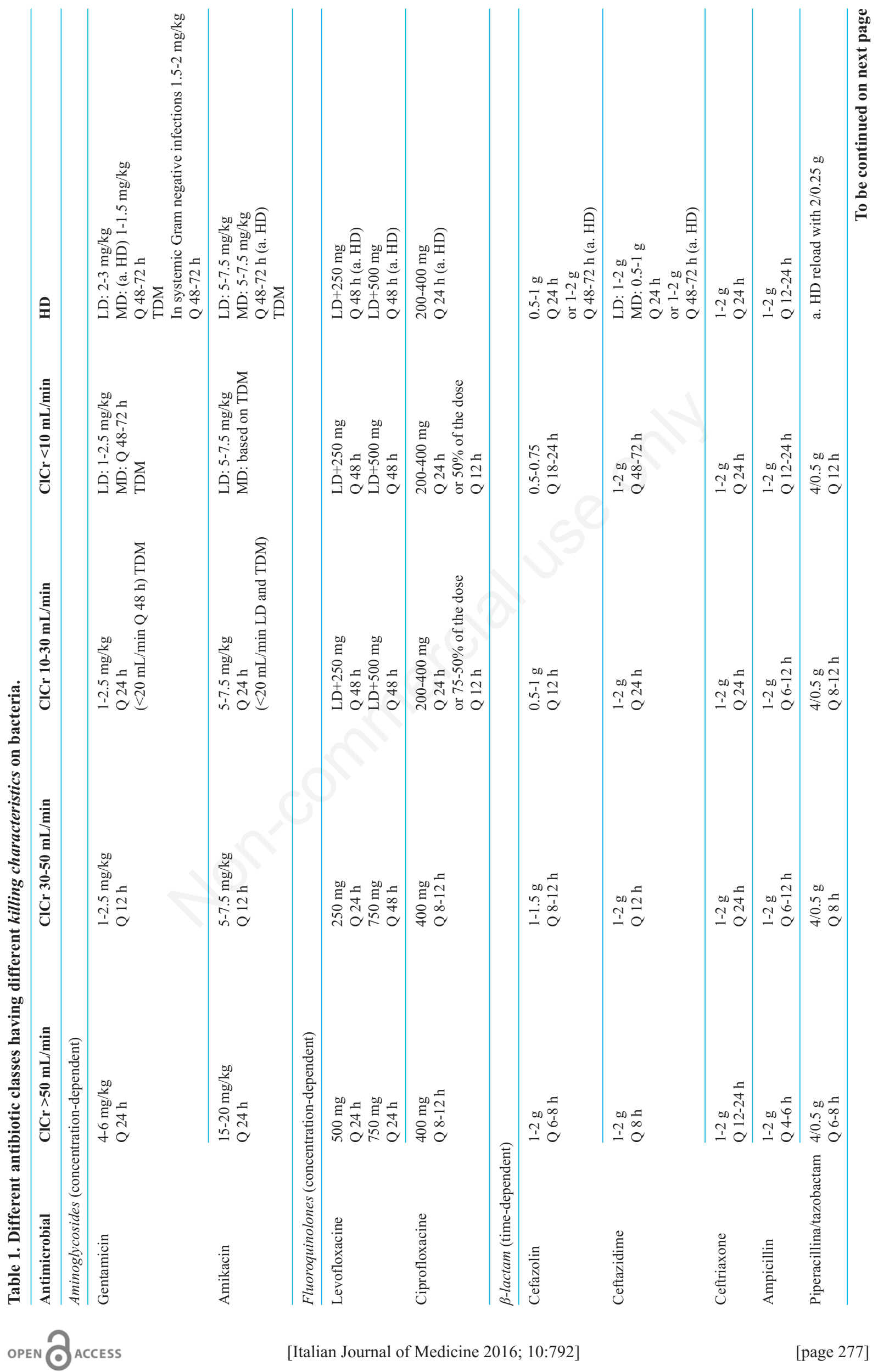




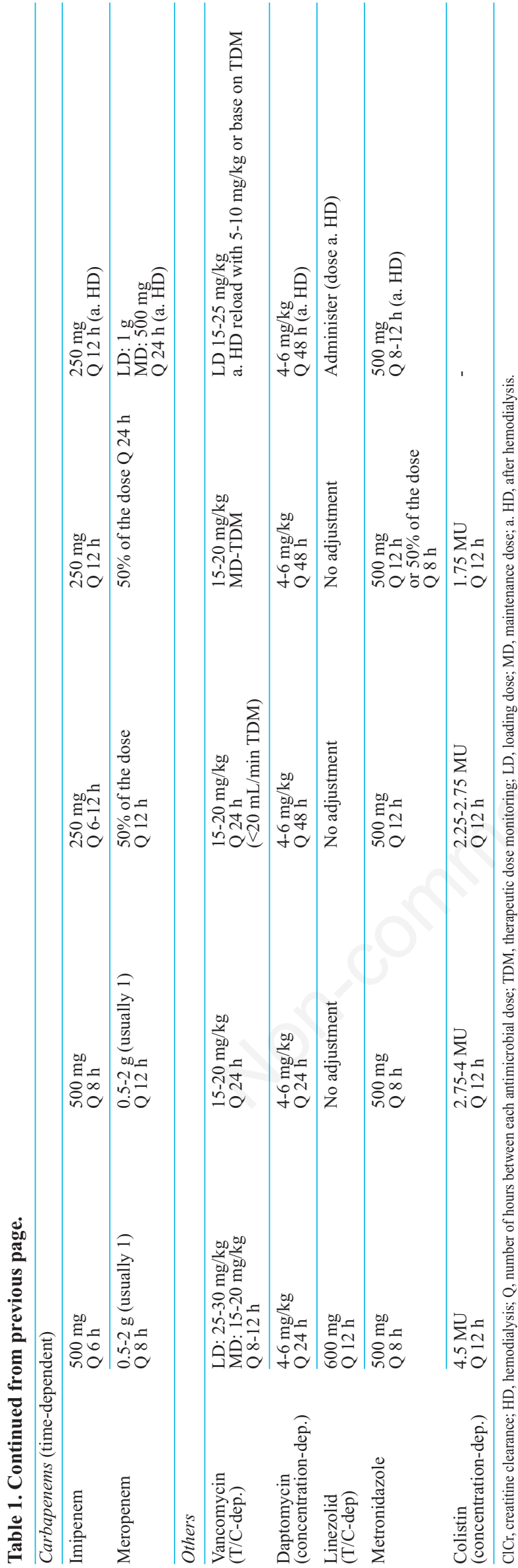

\section{Changes in antibiotic half-life}

Drug elimination half-life (T1/2) is directly related to antibiotic CL and Vd. In septic patients, there is often a hyperdynamic state with increased renal perfusion and consequently increased creatinine clearance and elimination of hydrophilic antibiotics, thus reducing their plasma half-life. Conversely, if the disease process advances, multiple organ dysfunction can lead to renal and/or hepatic failure determining a decreased CL and prolonged half-life with potentials for toxicity and drug accumulation.

In general, there are two important phases in the management of antibiotic therapy in critically ill septic patients. During the first day of therapy, frontloaded dosing, in spite of the presence of organ dysfunction, is required and must be guided by the predicted $\mathrm{Vd}$, which is likely increased due to fluid resuscitation and capillary leakage syndrome. From day 2, maintenance dosing can be adjusted in line with the CL associated with the organ dysfunction (renal and/or hepatic dysfunction).

Because of the great variability of organ function during a septic insult, therapeutic drug monitoring, whenever available, should be used to individualize dosing and ensure appropriate exposure to the antibiotic. ${ }^{14}$

\section{Renal replacement therapy}

Renal replacement therapy in its various forms can be continuous or intermittent and the clearance of the drug is unpredictable and inconstant causing wide variations in drugs concentrations. Achieving stable effective concentrations of antibiotics is a problematic issue, especially for time-dependent antibiotics where alternant clearance determines very high and very low concentrations (peaks and valley pattern) that expose the patient to the risk of toxicity and clinical failure.

Large molecules (>1000 Da) like vancomycin, are poorly cleared by hemodialysis, whereas smaller molecules (e.g., $\beta$-lactams and aminoglycosides) are largely cleared. Lipophilic antibiotics with a very large Vd, such as quinolones, are less represented in the vascular compartment and thus less available for clearance.

\section{Time is effectiveness and effectiveness is life}

The Surviving Sepsis Campaign (SSC) international consensus guidelines recommend initiating broad-spectrum antibiotic coverage within the first hour of recognition of severe sepsis and septic shock. ${ }^{3}$

These recommendations are based largely upon one large retrospective study published by Kumar et $a l$. in $2006^{4}$ and expert consensus.

In that study Kumar et al. reported that the administration of antibiotics within the first hour of docu- 
mented hypotension was associated with a survival rate of $79.9 \%$. Each hour of delay in antimicrobial administration over the ensuing 6 hours was associated with an average decrease in survival of $7.6 \%$ per hour. Despite these guidelines, data from the SSC registry demonstrated that, the practice of early antibiotic administration is still too low and only $68 \%$ of patients received broad-spectrum antibiotics within $3 \mathrm{~h}$ from Emergency Department presentation in a cohort of more than 15,000 patients. ${ }^{15}$

More recently, a meta-analysis of 11 studies including more than 16,000 patients published by Sterling et al. failed to show an increase in mortality in patients who received antibiotics more than 1 hour after severe sepsis/shock recognition. ${ }^{16}$

Moreover, there was no increasing mortality in the pooled odd ratios for each hourly delay from less than 1 to more than $5 \mathrm{~h}$ in antibiotic administration from severe sepsis/shock recognition. The authors were unable to demonstrate a significant mortality benefit of administering antibiotics within $3 \mathrm{~h}$ of emergency department triage or within 1 hour from shock recognition in severe sepsis and septic shock.

These results suggest that guideline-based timing for starting the therapy is not supported by robust evidence but this should not delay antibiotic administra- tion beyond the suggested timing indicated by current guidelines.

Early antibiotic administration with the right drug at the right dose, along with fluid resuscitation and infection source control, is still a cornerstone of treatment to improve survival in patients with severe septic syndromes.

\section{What does appropriate antimicrobial therapy mean and how to choose it}

Appropriate cultures should be obtained (if possible before antibiotics administration) but their obtainment should not delay the initiation of the therapy. Choosing the antibiotics should consider patient's history (e.g., allergies, recent antibiotics received ${ }^{17}$ ), comorbidities, clinical context (e.g., community- or hospital-acquired), Gram stain and local resistance data. ${ }^{3,18}$

Poor outcomes are associated with delays in initiating antimicrobial therapy as well as inadequate or inappropriate antibiotic regimens. ${ }^{5,19}$

The term appropriate antibiotic therapy refers to the use of initial antibiotic therapy concordant with guidelines specific for the clinical condition. In clinical studies, in the subset of patients with positive cul-

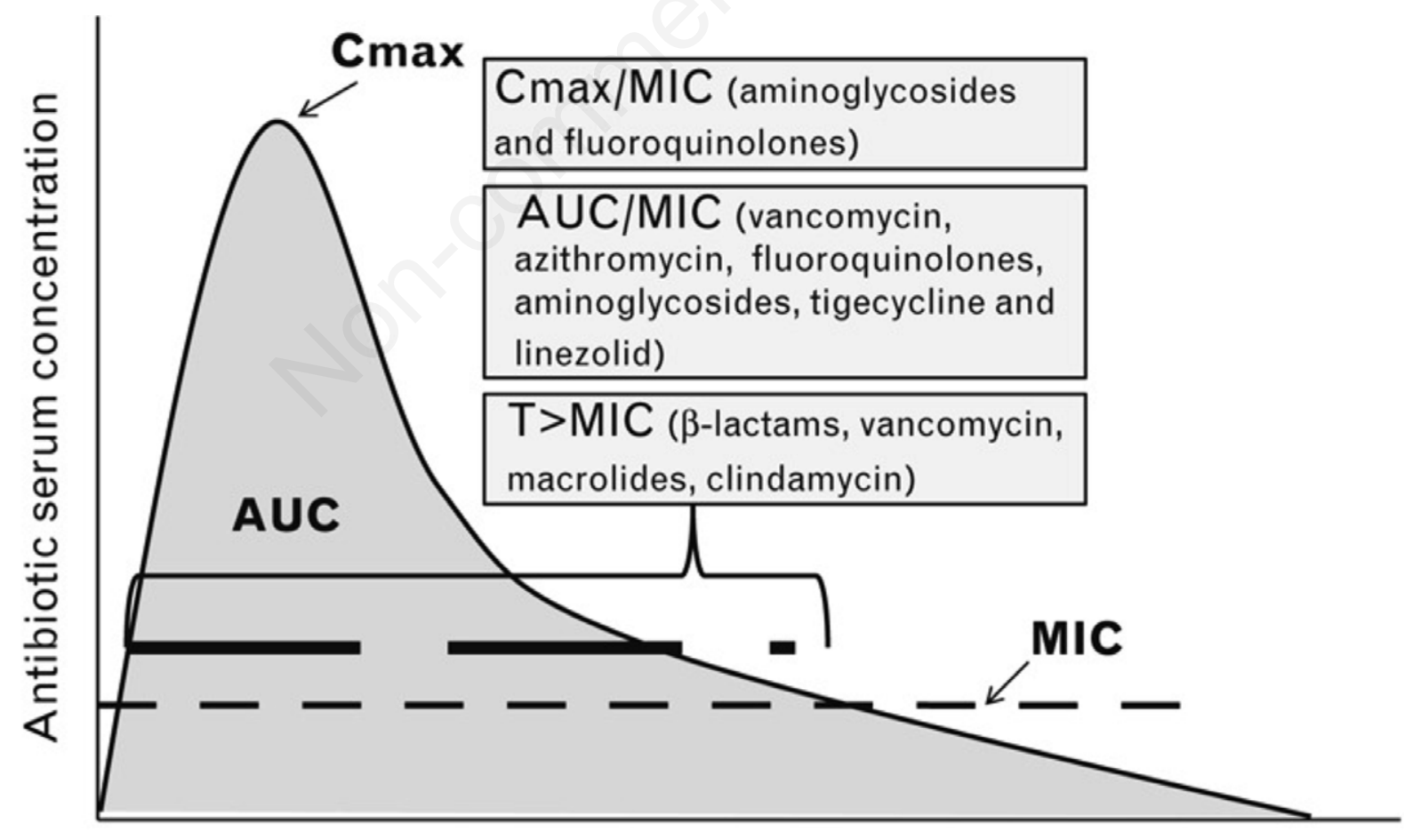

Time after antibiotic administration

Figure 2. Based on their killing characteristics antibiotics are distinguished in time-dependent, concentration-dependent and concentration-dependent antibiotics with time dependence. Cmax, maximal concentration; MIC, minimum inhibitory concentration; AUC, area under the curve. 
ture, appropriateness of antibiotic therapy refers also to the empirical choice of one or more antimicrobial agents to which the identified pathogen shows in vitro susceptibility.

Given the difficulty in choosing the initial therapy broad-spectrum coverage against both gram-positive and gram-negative should be considered when the potential pathogen or infection source in not fully clear.

There has been debate since many years regarding the potential benefits of combination versus monotherapy in the empiric treatment of infection in the critically ill patient.

In a meta-analysis by Kumar et al. including 50 studies, a survival benefit with combination therapy has been shown for those more severely ill patients. ${ }^{20}$

Support to the use of combination therapy relies mainly on expanding the coverage spectrum on multiple bacteria (especially in health-care associated infections) and the in vitro synergistic effect on bacterial killing. The debate is still in place, since clinical studies were not able to demonstrate the superiority of synergistic effect on clinical outcomes. ${ }^{21-23}$ Other potential disadvantages of combination therapy mainly consist of increasing toxicity, costs and possible emergence of multiple resistance and risk of bacterial and fungal superinfections. ${ }^{24}$

Current guidelines suggest empirical combination therapy with two or more antibacterial agents for neutropenic patients, for those at risk of multiple drugs resistant pathogens and for those with severe pneumonia and septic shock. ${ }^{3}$

It should be well kept in mind that, as soon as identification of the etiologic agent is available, prompt deescalation to the most effective agent (possibly with a single drug regimen) should be performed in order to reduce pressure on emergence of multiple resistance, risk of superinfections, adverse effects and costs.

Staphylococcus aureus is associated with significant morbidity if not treated early in the course of infection. Methicillin-resistant S. aureus (MRSA) now is a cause of sepsis not only in hospitalized patients, but also in community dwelling individuals without recent hospitalization ${ }^{25}$ so it is reasonable to provide coverage with intravenous vancomycin until the possibility of MRSA has been excluded. Alternative agents (e.g., daptomycin for non-pulmonary MRSA, linezolid, ceftaroline, ceftobiprole) should be considered for patients with refractory or virulent MRSA, or a contraindication to vancomycin.

If the probability of Pseudomonas is low vancomycin may be combined with a $3^{\text {rd }}$ or $4^{\text {th }}$ generation cephalosporin or a $\beta$-lactam $/ \beta$-lactamase inhibitor or a carbapenem.

Conversely, if Pseudomonas is a possible pathogen, vancomycin should be associated with two of the following agents: i) antipseudomonal cephalosporin; ii) antipseudomonal carbapenem; iii) antipseudomonal $\beta$ lactam/ $\beta$-lactamase inhibitor; iv) fluoroquinolone with good antipseudomonal activity (e.g., ciprofloxacin); v) aminoglycoside; vi) monobactam (e.g., aztreonam).

Selection of two agents from the same class should be avoided and antibiotic therapy should be tailored on the base of culture results and antimicrobial susceptibility data, even if the initial regimen has lead to clinical improvement.

The duration of therapy is typically 7 to 10 days (longer courses may be appropriate in patients who have slow clinical response, unremovable focus of infection, or immunologic deficiencies ${ }^{3}$. In neutropenic patients, antibiotic treatment should continue until the neutropenia has resolved or the planned antibiotic course is complete.

Patients in whom infection has been thoroughly excluded, should have antimicrobial therapy withdrawn to minimize colonization or infection with drug-resistant microorganisms and superinfection with other pathogens.

\section{Conclusions}

Very early antibiotic administration is the mainstay of treatment of severe sepsis syndromes, along with fluid resuscitation, advanced support, and source control. Based on pathophysiology of sepsis, pharmacodynamic and pharmacokinetic's principles should be well kept in mind when choosing antibacterial treatment. The old and famous refrain by the German microbiologist Paul Ehrlich hit fast and hit hard is still true after more than a century. Comprehensive clinical reasoning on the better antibiotic strategy is needed to offer the most appropriate treatment to an individual patient. Optimization of antibiotic therapy (i.e., modification of the drug or de-escalation) whenever results of cultures are available is recommended to enhance the potential of cure, and to reduce unnecessary exposure to inadequate drugs and decrease costs.

\section{References}

1. Rohde JM, Odden AJ, Kuhn L, et al. The epidemiology of acute organ system dysfunction from severe sepsis outside the intensive care unit. J Hosp Med 2013;8:121-32.

2. Mazzone A, Dentali F, La Regina M, et al. Clinical features, short-term mortality, and prognostic risk factors of septic patients admitted to internal medicine units: results of an Italian Multicenter Prospective Study. Medicine 2016;95:e2124.

3. Dellinger RP, Levy MM, Rhodes A, et al. Surviving Sepsis Campaign: international guidelines for management of severe sepsis and septic shock, 2012. Intensive Care Med 2013;39:165-228.

4. Kumar A, Roberts D, Wood KE, et al. Duration of hypotension before initiation of effective antimicrobial 
therapy is the critical determinant of survival in human septic shock. Crit Care Med 2006;34:1589-96.

5. Kumar A, Ellis P, Arabi Y, et al. Initiation of inappropriate antimicrobial therapy results in a fivefold reduction of survival in human septic shock. Chest 2009;136:1237-48.

6. Ebert SC, Craig WA. Pharmacodynamic properties of antibiotics: application to drug monitoring and dosage regimen design. Infect Control Hosp Epidemiol 1990;11: 319-26.

7. Taccone FS, Laterre PF, Dugernier T, et al. Insufficient beta-lactam concentrations in the early phase of severe sepsis and septic shock. Crit Care 2010;14:R126.

8. Roberts JA, Ulldemolins M, Roberts MS, et al. Therapeutic drug monitoring of beta-lactams in critically ill patients: proof of concept. Int Antimicrob Agents 2010; 36:332-9.

9. De Paepe P, Belpaire FM, Buylaert WA. Pharmacokinetic and pharmacodynamic considerations when treating patients with sepsis and septic shock. Clin Pharmacokinet 2002;41:1135-51.

10. Blot SI, Pea F, Lipman J. The effect of pathophysiology on pharmacokinetics in the critically ill patient-concepts appraised by the example of antimicrobial agents. Adv Drug Deliv Rev 2014;77:3-11.

11. Roberts JA, Abdul-Aziz MH, Lipman J, et al. Individualised antibiotic dosing for patients who are critically ill: challenges and potential solutions. Lancet Infect Dis 2014; 14:498-509.

12. Felton TW, Hope WW, Roberts JA. How severe is antibiotic pharmacokinetic variability in critically ill patients and what can be done about it? Diagn Microbiol Infect Dis 2014;79:441-7.

13. Udy AA, Baptista JP, Lim NL, et al. Augmented renal clearance in the ICU: results of a multi center observational study of renal function in critically ill patients with normal plasma creatinine concentrations. Crit Care Med 2014;42:520-7.

14. Ulldemolins M, Roberts JA, Lipman J, Rello J. Antibiotic dosing in multiple organ dysfunction syndrome. Chest 2011;139:1210-20.

15. Levy M, Dellinger R, Townsend S, et al. The Surviving
Sepsis Campaign: results of an international guidelinebased performance improvement program targeting severe sepsis. Crit Care Med 2010;38:367-74.

16. Sterling SA, Miller WR, Pryor J, et al. The impact of timing of antibiotics on outcomes in severe sepsis and septic shock: a systematic review and meta-analysis. Crit Care 2015;43:1907-15.

17. Johnson MT, Reichley R, Hoppe-Bauer J, et al. Impact of previous antibiotic therapy on outcome of Gram-negative severe sepsis. Crit Care Med 2011;39:1859.

18. Sibbald WJ, Vincent JL. Round table conference on clinical trials for the treatment of sepsis. Crit Care Med 1995;23:394.

19. Garnacho-Montero J, Garcia-Garmendia JL, BarreroAlmodovar A, et al. Impact of adequate empirical antibiotic therapy on the outcome of patients admitted to the intensive care unit with sepsis. Crit Care Med 2003;31:2742.

20. Kumar A, Safdar N, Kethireddy S, Chateau D. A survival benefit ofcombination antibiotic therapy for serious infections associated with sepsisand septic shock is contingent only on the risk of death: a meta-analytic/metaregression study. Crit Care Med 2010;38:1651-64.

21. Leibovici L, Paul M, Poznanski O, et al. Monotherapy versus beta-lactam-aminoglycoside combination treatment for gram-negative bacteremia: a prospective, observational study. Antimicrob Agents Chemother 1997; 41:1127.

22. Rubinstein E, Lode H, Grassi C. Ceftazidimemonotherapy vs. ceftriaxone/tobramycin for serious hospital-acquired gram-negative infections. AntibioticStudy Group. Clin Infect Dis 1995;20:1217.

23. Paul M, Lador A, Grozinsky-Glasberg S, Leibovici L. Beta lactam antibiotic monotherapy versus beta lactamaminoglycoside antibiotic combination therapy for sepsis. Cochrane Database Syst Rev 2014;(1):CD003344.

24. Vincent JL, Bassetti M, Francois B, et al. Advances in antibiotic therapy in the critically ill. Crit Care 2016;20:133.

25. Fridkin SK, Hageman JC, Morrison M, et al. Methicillin-resistant Staphylococcus aureus disease in three communities. N Engl J Med 2005;352:1436. 